\title{
A latent variable approach to heat load prediction in thermal grids ${ }^{\star}$
}

\author{
Johan Simonsson ${ }^{1,2, \dagger}$, Khalid Tourkey Atta ${ }^{1}$, Dave Zachariah $^{3}$, Wolfgang Birk ${ }^{1}$
}

\begin{abstract}
In this paper a new method for heat load prediction in district energy systems is proposed. The method uses a nominal model for the prediction of the outdoor temperature dependent space heating load, and a data driven latent variable model to predict the time dependent residual heat load. The residual heat load arises mainly from time dependent operation of space heating and ventilation, and domestic hot water production. The resulting model is recursively updated on the basis of a hyper-parameter free implementation that results in a parsimonious model allowing for high computational performance. The approach is applied to a single multi-dwelling building in Luleå, Sweden, predicting the heat load using a relatively small number of model parameters and easily obtained measurements. The results are compared with predictions using an artificial neural network, showing that the proposed method achieves better prediction accuracy for the validation case. Additionally, the proposed methods exhibits explainable behavior through the use of an interpretable physical model.
\end{abstract}

\section{INTRODUCTION}

District heating and cooling systems are essential technologies towards reaching climate goals and rendering an expected growth of district heating capacity in Europe [1], [2]. Moreover, 4th generation district heating networks $(4 \mathrm{GDH})[3]$, with lower supply temperatures generated from a wide variety of energy sources including renewable energy sources and waste heat utilization, requires novel control schemes and has ultimately increased the demand for dynamic simulation of district heating grids. In the Digital Roadmap for District Heating and Cooling [4], the digital twin, a simulation model with embedded intelligence that is updated alongside the process, is identified as an important tool for this new generation of district heating networks.

Within this scope, accurate and computationally efficient heat load predictions for consumers are crucial. District heating grids often range over thousands of consumers, and better computationally performance means a higher resolution can be used in the grid simulation. Common use cases for a digital twin include, but are not limited to, design of new district heating grids or subnetworks, design of novel control and operation schemes, and production scheduling optimization.

Prediction of heat load for district heating consumers is not new and has been investigated by several studies, e.g using seasonal dynamic models such as SARIMAX [5] and

\footnotetext{
${ }^{1}$ Control Engineering Group, Luleå University of Technology, Sweden ${ }^{2}$ Optimation AB, Uppsala, Sweden

${ }^{3}$ Department of Information Technology, Uppsala University, Sweden

${ }^{\dagger}$ Corresponding author: johan.simonsson@ltu.se

* The authors want to thank Luleå Energi $\mathrm{AB}$ and especially Fredrik Udén for discussions and making data available. This study was financially supported by the Swedish Energy Agency under grant 43090-2, Cloudberry Datacenters.
}

by artificial neural networks (ANN) [6], [7]. Models using separation of heating and domestic tap water consumption heat load has been thoroughly covered in [8], and usage for prediction of a single house using has been covered in [9]. It should be noted that single buildings, in general, show more erratic patterns of consumption than the total consumption in the grid, and that the same level of prediction accuracy can not be reached. A comprehensive overview of predictive methods for district heating load can be found in [10].

In the present study, a heat load prediction model is proposed, using a nominal model for prediction of outdoor temperature dependent space heating load, and a latent variable model for the residual heat load, where the model is updated simultaneously and recursively. The theoretical framework is introduced in [11] and adapted to suit the district heating heat load prediction use case. The model is verified against a single multi-dwelling building in Luleå, Sweden.

The prediction accuracy is compared to a neural network approach, with the latent variable method showing a higher prediction accuracy for the test case. Compared to using artificial neural networks, the latent variable method allows for explainability due to the composition of the model. Here, the nominal model represents the physical building and substation, and the data driven latent variable part represents the time dependent user and control behavior. Compared to SARIMAX and more specialized models also using a split between heat load used for hot tap water consumption and space heating load, as described in [8], the presented method produces a model that is recursively and simultaneously updated for the split model, is straight forward to aggregate for simulating larger groups of buildings, and requires less parameters.

The paper is organized as follows. The first section provide the general structure of the model and a motivation for it. The following two sections introduce the nominal model that is used to represent the thermal behavior of the building, and the latent variable model. Therein, the latent variable model for the residual heat is discussed in detail. The results from the test case are given together with the comparison with the ANN model in the Result section. The paper ends with some conclusions and outlook.

\section{General StRuCture}

The observed heat load is the output of the substation control system including domestic hot water production, in conjunction with the thermal grid. The substation control strategy is generally not known for the entire grid, and the hot tap water use is largely stochastic in its nature. However, 
by using appropriate covariates we can identify the most commonly used control strategies for district heating. Here, a hybrid model approach, described in detail in [11], is used where $\hat{y}_{\text {nom }}(t)=\Theta \varphi(t)$ is a predictive model for the outdoor temperature dependent nominal heat load, mainly space heating. The residual load, mainly predicting time dependent space heating and ventilation, and domestic hot water production, $\varepsilon(t)=y(t)-\hat{y}_{n o m}(t)$ is then assumed to be gaussian

$$
\varepsilon(t) \sim \mathcal{N}(Z \gamma(t), \Sigma)
$$

with a time-varying expected value $Z \gamma(t)$, where the latent variable $Z$ and covariance matrix $\Sigma$ is unknown. The total predicted heat load is consequently

$$
\hat{y}(t)=\hat{y}_{n o m}(t)+\hat{y}_{r e s}(t)=\Theta \varphi(t)+Z \gamma(t) .
$$

The split model serves three purposes. For one, the nominal model is assumed to have a long term dynamic component due to possible feedback and the thermal inertia of the building, whereas we for computation efficiency do not desire to include these time lags in the user behaviordependent part. Second, by using a suitable basis function $\gamma(t)$ it is possible to approximate both periodic and non periodic time dependent behavior without pre-processing of the data. Third, the explicit separation of outdoor temperature dependent and time dependent behavior allows for physical interpretability of the nominal model that can be exploited in future separate categorizations of the two when a larger building stock is targeted.

Joint estimation of the nominal model and the residual load model is based on the maximum likelihood approach with a latent variable $Z$ that is distributed as

$$
\operatorname{vec}(Z) \sim \mathcal{N}(0, D)
$$

The nominal model parameters $\Theta$ are found by the maximum likelihood approach, maximizing

$$
p(Y \mid \Omega)=\int p(Y \mid \Omega, Z) p(Z) d z
$$

where $\Omega=\{\Theta, D, \Sigma\}$. The problem may have local minima, and an estimation of the parameters is found using the Expectation Maximization technique [12] where the cost function obtained from the maximization (4) is guaranteed to decrease monotonically. The latent variables $\mathrm{Z}$ can then be estimated at the optimal estimate of $\Omega$. The calculations include a data-adaptive regularizing term that produces parsimonious estimates of Z [13] without user-specified hyperparameters.

Notably, the nominal model must be linear in the parameters, but nonlinear inputs can be provided if applicable. As the total heat load for the grid is predicted as the sum of distributed loads it allows for the use of specific models for consumers that cannot be predicted from readily available data, e.g. certain types of industrial plants. For the individual consumer heat load prediction, the following information is updated and stored for every time step

- $\Theta$ matrix - Parameters of the nominal model
- Z matrix - Parameters of the latent variable model

- $\Sigma$ matrix - Variance of the total model error

- $\mathrm{n}$ - Number of observations used for the model

The aggregation of distributed heat loads is straightforward since the total predicted heat load at a given time instance is the sum of individual predictions. The errors are uncorrelated, and the total predicted heat load for $N$ consumers and the total variance is given by:

$$
\hat{y}_{\mathrm{tot}}(t)=\sum_{i=1}^{N} \hat{y}_{i}(t) \quad \text { and } \quad \Sigma_{\mathrm{tot}}=\sum_{i=1}^{N} \Sigma_{i},
$$

which can be learned recursively.

\section{NOMINAL MODEL}

The space heating load is often mainly outdoor temperature dependent due to the most common control strategies, involving possible combinations of outdoor temperature dependent supply temperature from the grid operator, outdoor temperature dependent feed forward control for the substation, and indoor temperature feedback control for the substation. The latter is indirectly affected by the outdoor temperature. The control strategies can also directly or indirectly (for feedback control) depend on factors such as wind speed, solar radiation and precipitation.

The goal of the space heating control strategy is generally to keep the indoor temperature at a stable comfort temperature. However, by manipulating the stored heat in the building by raising or lowering the indoor temperature slightly, the building can be used as a passive thermal storage for the grid [14].

A simplified energy balance for the building adapted from [15] describes the dynamics of the building

$$
C_{t h} \frac{\partial T_{b}(t)}{\partial t}=Q_{\mathrm{sh}}(t)+Q_{v}(t)+Q_{\mathrm{int}}(t)-Q_{\mathrm{out}}(t)
$$

where $Q_{\mathrm{sh}}(t)$ is the space heating load that we want to predict with the nominal model, $C_{\mathrm{th}}$ is the thermal mass of the building, $T_{b}(t)$ the lumped temperature of the building, $Q_{v}(t)$ is heat flow from ventilation air, $Q_{\text {int }}(t)$ the energy from e.g. electrical equipment and residents, and $Q_{\text {out }}(t)$ the temperature losses to ambient. The temperature losses to ambient can be approximated [16] with a linear function of the outdoor temperature and the lumped building temperature

$$
Q_{\text {out }}(t)=k_{h t} A_{h t}\left(T_{b}(t)-T_{o}(t)\right) .
$$

If the supply temperature or substation only uses a feed forward based on the outdoor temperature, identification of the outdoor temperature dependency of the heat load is straight forward $Q_{\mathrm{sh}}(t)=\theta_{1} T_{o}(t)$ under the assumption that the feed forward curve is reasonably linear. For the feedback case we can view the system as the block diagram shown in Figure 1 .

If we first consider the blocks within the dotted line in Figure 1, in Laplace form we have the building temperature

$$
\begin{array}{r}
T_{b}(s)=\left(Q_{s h}(s)+V(s)\right. \\
\left.+k_{h t} A_{h t}\left(T_{b}(s)-T_{o}(s)\right)\right) G_{b}(s)
\end{array}
$$




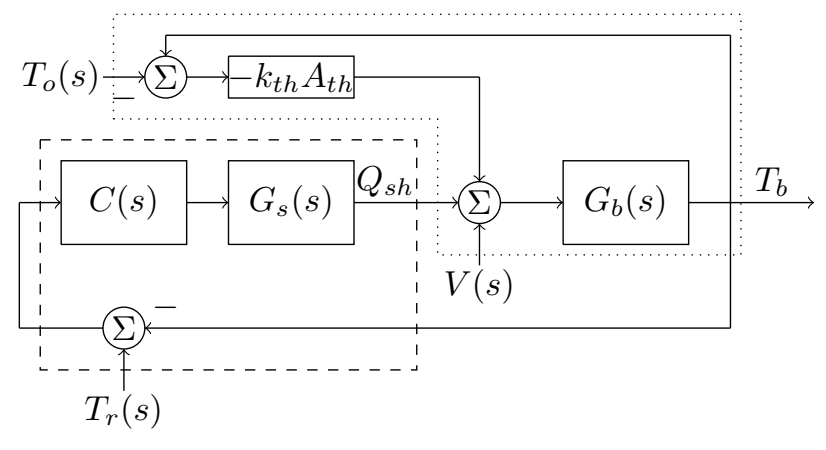

Fig. 1. Block diagram of the feedback system.

that we can rearrange to get

$$
T_{b}(s)=\tilde{G}(s)\left(Q_{s h}(s)+V(s)+k_{h t} A_{h t} T_{o}(s)\right)
$$

where, by inserting (6) in Laplace form, we have

$$
\tilde{G}(s)=\frac{G_{b}(s)}{\left(1+k_{h t} A_{h t} G_{b}(s)\right)}=\frac{1}{k_{h t} A_{h t}+C_{t h} s} .
$$

The substation dynamics are fast compared to the building dynamics, so we approximate the blocks within the dashed line in Figure 1 as $\tilde{C}(s)=C(s) G_{s}(s)$. We have thus simplified the block diagram from Figure 1 to the form seen in Figure 2.

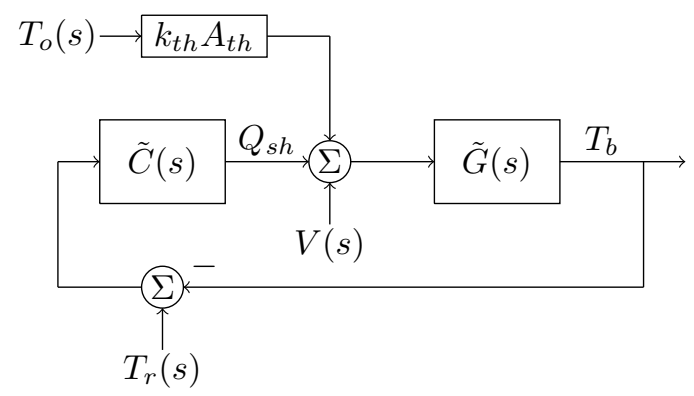

Fig. 2. Simplified representation of the feedback system.

We can now look at $Q_{\text {sh }}$ that is our target quantity for the prediction, once again in Laplace form

$$
\begin{array}{r}
Q_{s h}(s)=\tilde{C}(s) \tilde{G}(s)\left(T_{r}(s)-\left(Q_{s h}(s)\right.\right. \\
\left.\left.+k_{h t} A_{h t} T_{o}(s)+V(s)\right)\right),
\end{array}
$$

where rearranging the equation gives us three parts

$$
\begin{array}{r}
Q_{s h}(s)=\underbrace{-\frac{\tilde{C}(s) \tilde{G}(s)}{1+\tilde{C}(s) \tilde{G}(s)} V(s)}_{Q_{s h}^{R}(s)} \\
-\underbrace{\frac{k_{h t} A_{h t} \tilde{C}(s) \tilde{G}(s)}{1+\tilde{C}(s) \tilde{G}(s)}}_{Q_{s h}^{T}(s)} T_{o}(s)+\frac{\tilde{C}(s)}{1+\tilde{C}(s) \tilde{G}(s)} T_{r}(s) .
\end{array}
$$

Here, $Q_{s h}^{T}(s)$ is the outdoor temperature dependent space heating load, that is target quantity for the nominal model.
The set point of the indoor temperature $T_{r}(s)$ can be assumed to be constant in relation to the outdoor temperature $T_{o}(s)$, and the building temperature $T_{b}(s)$ can be assumed to be close to the reference temperature $T_{r}(s)$ so that the third term is relatively small. We then leave the prediction of $Q_{s h}^{R}$ as a time dependent residual part that is modeled by the latent variable model. The most common approach for feedback control of the indoor temperature is a PID controller, so that we get

$$
\tilde{C}(s)=K_{p}+\frac{K_{i}}{s}+K_{d} s
$$

where inserting (13) and (10) into $Q_{s h}^{T}(s)$ from the equation above we get

$$
\begin{gathered}
Q_{s h}^{T}(s)= \\
\frac{-k_{h t} A_{h t}\left(K_{i}+K_{p} s+K_{d} s^{2}\right)}{K_{i}+\left(k_{h t} A_{h t}+K_{p}\right) s+\left(C_{t h}+K_{d}\right) s^{2}} T_{o}(s)
\end{gathered}
$$

that can be approximated by an ARX model of sufficiently high order. Further, exploiting the knowledge that there is generally no space heating when the outdoor temperature is high enough, we can substitute the outdoor temperature with $\Delta T(t)=\max \left(T_{c}-T_{o}(t), 0\right)$ where $T_{c}$ is a user specified threshold temperature, to avoid the discontinuity. The nominal model can then be written as

$$
\hat{y}_{\mathrm{nom}}(t)=\Theta \varphi(t)=\Theta\left[\begin{array}{c}
1 \\
\Delta T(t) \\
\Delta T(t-1) \\
\vdots \\
\Delta T\left(t-n_{b}\right)
\end{array}\right] .
$$

Using $n_{b}=24$, that is $24 \mathrm{~h}$ of time lags, has shown to give good predictions even during sharp outdoor temperature gradients for the building and substation used in the examples. Results show that including precipitation, wind speed or sun radiation has a negligible impact on the prediction accuracy of the example building. However, this is influenced by the control strategy in conjunction with factors such as where the building is located geographically, if it is shielded from wind, and the isolation of the building. Luleå is also located in the north of Sweden where the sun radiation during winter is low, whereas in the summer when the sun radiation is high there is no need for space heating.

\section{LATENT VARIABLE MODEL}

The residual heat load that is predicted by the latent variable model is

$$
y_{\text {res }}(t)=Q_{s h}^{R}(t)+Q_{t w}(t)
$$

including the residual part from (12), and $Q_{t w}(t)$ that is the heat used for domestic hot water production. At the substation level it is concluded in [17] that there exists four main heat load patterns based on the most commonly used control strategies for radiator and ventilation systems: continuous operation control, night set-back control, time 
clock operation control 5 days a week and time clock operation control 7 days a week.

The hot tap water heat load patterns are highly dependent on the type of building, where a school is expected to have distinctively different hot tap water consumption patterns than a multi-dwelling apartment building. However, these patterns tend to show periodic behavior depending on time dependent covariates such as time of the day, day of the week, period of the year and weekend / not weekend, something that also holds for the time dependent control strategies above. These covariates are used as input $u_{\gamma}(t)$ to the latent variable model.

The binary and continuous or periodic inputs to the latent variable model are denoted $u_{\gamma b}(t)$ and $u_{\gamma p}(t)$, with sizes $n_{\gamma b}$ and $n_{\gamma p}$ respectively. By using a Fourier expansion of $u_{\gamma}(t)$ as input to the latent variable model we can fit both periodic and continuous behavior from the covariates. Periodic and continuous signals are then approximated by the vector $\gamma_{p}(t)$ of orthogonal Fourier series basis expansions up to $M$ harmonics. Using the time of day expressed in hours $t_{d}(t)$, day of week $d_{w}(t)$, and week number $w_{y}(t)$ for the periodic or continuous part, and weekend/not weekend $w k(t)$ and summer/not summer $s(t)$ as a binary inputs, we get

$$
u_{\gamma p}(t)=\left[\begin{array}{c}
t_{d}(t) \\
d_{w}(t) \\
w_{y}(t)
\end{array}\right] \quad \text { and } \quad u_{\gamma b}(t)=\left[\begin{array}{c}
w k(t) \\
s(t)
\end{array}\right] .
$$

where the weekend signal includes official holidays, and May-August are considered as summer months. The basis expansion for each input of $u_{\gamma p, i}(t)$ and basis number $j$ can be written as

$$
b_{i, j}(t)=\left[\begin{array}{l}
\cos \left(\frac{j \pi u_{\gamma p, i}(t)}{2 \ell_{i}}\right) \\
\sin \left(\frac{j \pi u_{\gamma p, i}(t)}{2 \ell_{i}}\right)
\end{array}\right]
$$

where $\ell_{i}$ is the boundary for each input. The $\gamma_{p}(t)$ vector contains the basis expansions of the periodic inputs

$$
\gamma_{p}(t)=\left[\begin{array}{c}
b_{1,1}(t) \\
\vdots \\
b_{1, M}(t) \\
b_{2,1}(t) \\
\vdots \\
b_{n_{\gamma p}, M}(t)
\end{array}\right] .
$$

For the binary variables we have

$$
\gamma_{b}(t)=\left[\begin{array}{c}
1 \\
u_{\gamma b, 1}(t) \\
\left(1-u_{\gamma b, 1}(t)\right)
\end{array}\right] \otimes \ldots \otimes\left[\begin{array}{c}
1 \\
u_{\gamma b, n \gamma b}(t) \\
\left(1-u_{\gamma b, n \gamma b}(t)\right)
\end{array}\right]
$$

where $\otimes$ denotes the Kronecker product, and finally

$$
\gamma(t)=\left(\left[\begin{array}{ll}
\mathbf{0}_{B} & I_{B}
\end{array}\right] \gamma_{b}(t)\right) \otimes \gamma_{p}(t)
$$

where $\mathbf{0}_{B}$ and $\mathbf{I}_{B}$ are $n_{b} \times 1$ and $n_{b} \times n_{b}$ respectively, with $n_{b}=\left((2+1)^{n_{\gamma b}}-1\right)$. The latent variable model will then have $n_{\gamma}=2 M n_{\gamma p}\left((2+1)^{n_{\gamma b}}-1\right)$ variables, where in the results section we have used $M=8$ and thus 128 variables in $Z$. For the case study including cross-terms of $\gamma(t)$ have not provided better prediction accuracy and have been left out for higher computational performance.

\section{RESULTS}

Hourly heat load measurements were acquired from a single multi-dwelling building connected to the district heating network of Luleå, Sweden. The data was provided by Luleå Energi $\mathrm{AB}$ and anonymized in compliance with the General Data Protection Regulation (GDPR). Furthermore, weather data from the Swedish Metereological Institute (SMHI) and temporal data such as date and time was used. The heat load measurement has a dead band of $10 \mathrm{~kW}$ causing a quantization effect on the signal, where the percentage relative heat load can be seen in Figure 3.

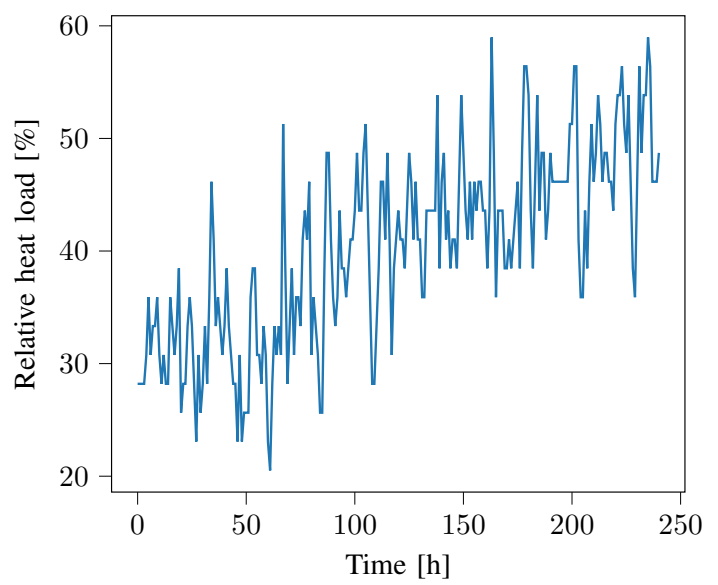

Fig. 3. Sample of heat load data series.

The model has been trained with one year of measurement data, and is then continuously predicting $24 \mathrm{~h}$ ahead out of sample for the following year, updating the model for every step ahead (walk-forward prediction). Each sample in the plot is the $24 \mathrm{~h}$ ahead prediction. Predictions use the actual temperature ahead, where in real life weather predictions needs to be used.

As a reference for the prediction accuracy a feed forward Artificial Neural Network (ANN) has been used. For a single hidden layer the ANN can be represented [7] as

$$
f(x)=\sum_{j=1}^{N} w_{j} \psi_{j}\left[\sum_{i=1}^{M} w_{i j} x_{i}+w_{i o}\right]+w_{j o}
$$

where $M$ is the number of inputs, $N$ the number of hidden units and $\psi$ the transfer function for each hidden unit. The mean square error (MSE) has been used as cost function, and the $\tanh (\mathrm{x})$ as activation function. The neural network has been implemented with 1-3 hidden layers, that has previously been shown to produce good prediction results in [7]. The results presented are from the best prediction, which in this case was using three layers. The ANN has been implemented using the MATLAB Deep Learning Toolbox. The same covariates as for the latent variable model have been used. 
In order to evaluate the results, the relative root mean square error (rRMSE) of the data has been used as a performance metric. Since this is a metric commonly used for time series prediction the results are comparable to other methods, and indeed show that the estimates are in line with what has been previously reported. The reported rRMSE is for the whole validation data set, including the summer months with low heat load.

$$
\operatorname{rRMSE}=\frac{1}{\bar{y}} \sqrt{\frac{1}{n} \sum_{i=1}^{n}\left(y_{i}-\hat{y}_{i}\right)^{2}}
$$

The resulting rRMSE\% can be seen in Table [] where the latent variable approach performs significantly better than the ANN method for this test case.

TABLE I

PREDiction, RELATIVE RMSE RESUlts

\begin{tabular}{c|c}
\hline LAVA Validation dataset & $18.2 \%$ \\
ANN Validation dataset & $28.8 \%$ \\
\hline
\end{tabular}

Two prediction plots are provided, one time period of 10 days with a relatively sharp temperature gradient, and one for a summer month without any space heating load. Prediction results can be seen in Figure 4 and 5, with the results from the ANN prediction as a reference. Notably, some very sharp morning peaks in load occur during some weekdays, but not other weekdays, nor the same weekdays the week after. Such irregularities in consumer behaviour can not be predicted from cyclic variables like the time of the day, and would need additional covariates for prediction, if they are at all predictable.

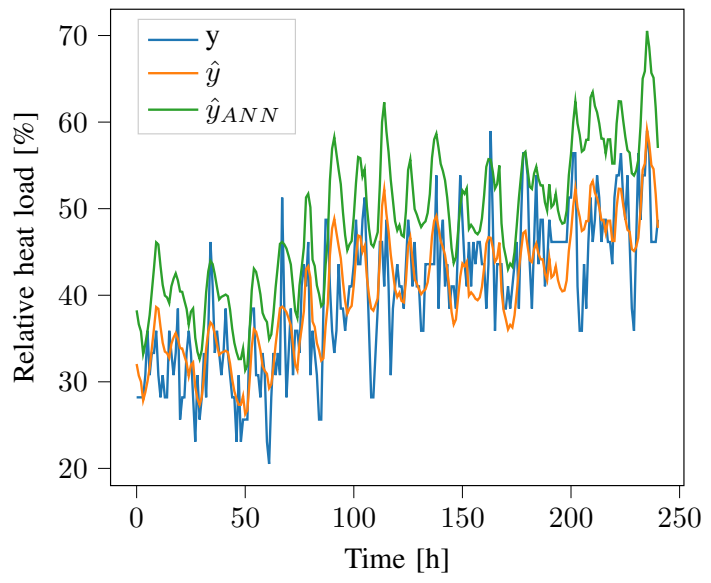

Fig. 4. 24h forecast and measured heat load for 10 winter days.

The output from the nominal model $\hat{y}_{n o m}(t)=\Theta \varphi(t)$ is seen in Figure 6 As expected the nominal model follows the $\Delta T$ temperature closely, but also has a dynamic component.

The output of the latent variable model $\hat{y}_{\text {res }}(t)=Z \gamma(t)$ that can be seen in Figure 7 shows that the models have adapted to the diurnal pattern of the heat load, with distinct different patterns for workdays and the weekend. The resulting $Z$ in this case has 70 non-zero parameters.

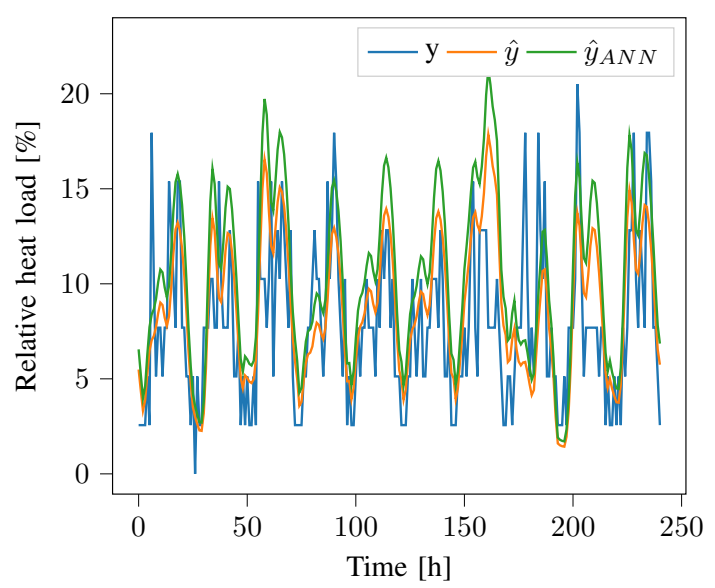

Fig. 5. 24h forecast and measured heat load for 10 summer days.

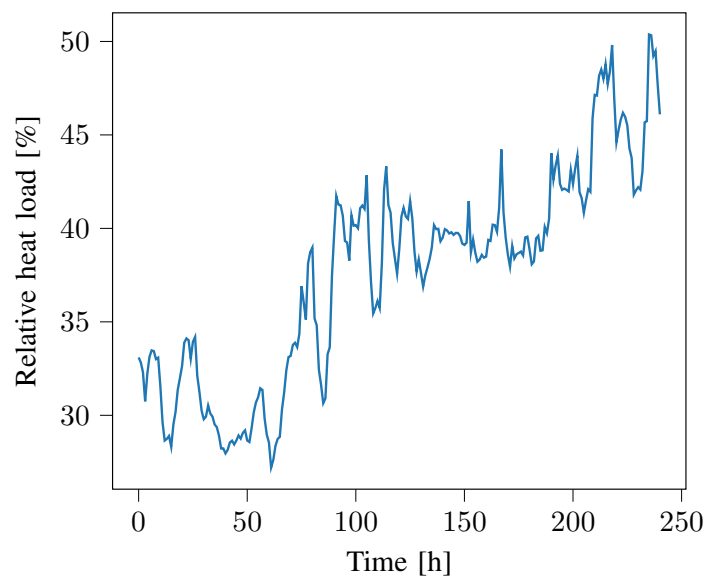

Fig. 6. Prediction contribution from the nominal model.

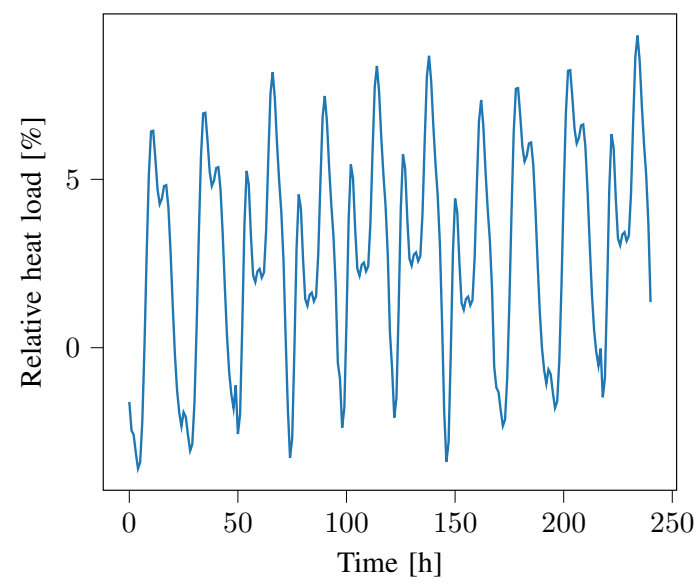

Fig. 7. Prediction contribution from the latent variable model, showing heat load patterns for both weekend and workday.

A comparison between the outputs from $\hat{y}_{\text {res }}(t)=Z \gamma(t)$ using different number of harmonics $M$ for the latent variable model can be seen in Figure 8, with the corresponding rRMSE metrics for the validation data set listed in Table I. Since more harmonics renders a larger $Z$ matrix this comes 
with a computational performance penalty.

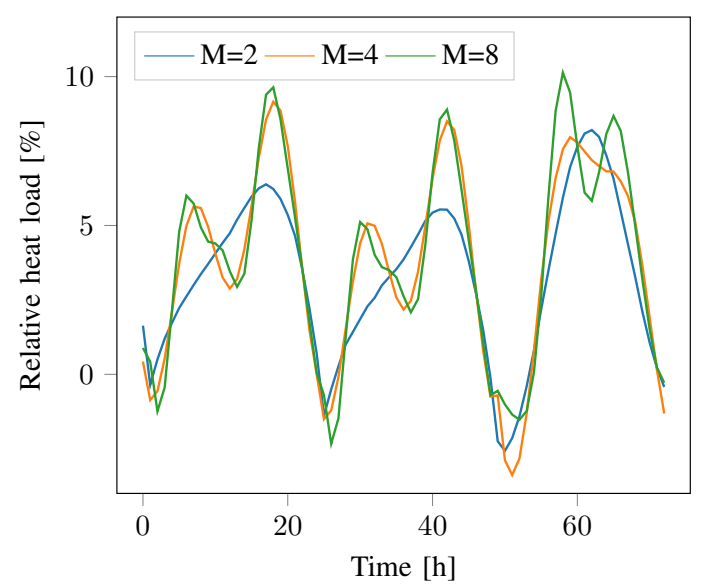

Fig. 8. Output from the latent variable model using different number of harmonics.

TABLE II

PREDICTION RELATIVE RMSE RESULTS

\begin{tabular}{c|c}
\hline $\mathrm{M}=2$ & $19.0 \%$ \\
$\mathrm{M}=4$ & $18.4 \%$ \\
$\mathrm{M}=8$ & $18.2 \%$ \\
$\mathrm{M}=12$ & $18.49 \%$ \\
\hline
\end{tabular}

Over the year the largest deviations from the actual load are typically found around certain holidays, suggesting that the workday-weekend split might not be fine grained enough for the most accurate predictions.

\section{CONCLUSIONS AND FUTURE RESEARCH}

In this article, it is shown how a hybrid approach using a nominal model for outdoor temperature dependent heat load together with a latent variable model for the residual heat load, can be used for prediction of the total heat load for a multi-dwelling building. The model structure is suitable both for offline simulation and in an online setup where the models are continuously updated. The implementation of the parameter estimation is recursive and hyper-parameter free, allowing for an easy parameterization of the model. The algorithm produces parsimonious models that can be efficiently simulated on a standard computer.

In order to simulate a city scale district heating network, with use cases such as the addition of new city quarters to the grid, further research on the classification of different types of buildings is needed. The proposed model structure allows for separate categorization of the nominal and latent variable model structures.

The model structure allows for a straight-forward aggregation of the distributed heat loads, whereas aggregation of consumers for a specific point in the network requires accounting for the distribution of the thermal heat.

For a city scale simulation, different models, such as models with more detailed dynamics, consumer dependency on other covariates, and consumers where the heat load cannot be predicted from acquirable signals, need to be integrated in the same simulation. Accordingly, a generic consumer heat load framework needs to be considered, where the proposed model is a piece of a larger puzzle.

\section{REFERENCES}

[1] B. Doračić, T. Novosel, T. Pukšec, and N. Duić, "Evaluation of excess heat utilization in district heating systems by implementing levelized cost of excess heat," Energies, vol. 11, no. 3, 2018.

[2] D. Connolly, B. V. Mathiesen, P. A. Østergaard, B. Möller, S. Nielsen, H. Lund, D. Trier, U. Persson, D. Nilsson, and S. Werner, "Heat Roadmap Europe 1: First Pre-Study for the EU27," 2012.

[3] H. Lund, S. Werner, R. Wiltshire, S. Svendsen, J. E. Thorsen, F. Hvelplund, and B. V. Mathiesen, "4th Generation District Heating (4GDH): Integrating smart thermal grids into future sustainable energy systems," Energy, vol. 68, pp. 1-11, Apr. 2014.

[4] R. de Beaufort, S. S. Jensen, C. Johansson, R. Hellmer, M. Jungic, V. Kaasinen, M. Karstoft, A. Schache, R.-R. Schmidt, P. Song, D. Vanhoudt, and X. Zhao, "Digital Roadmap for District Heating \& Cooling," 2018.

[5] S. Grosswindhager, A. Voigt, and M. Kozek, "Online Short-Term Forecast of System Heat Load in District Heating Networks," Proceedings of the 31st international symposium on forecasting, Prag, Czech Republic; 2011, p. 8, 2011.

[6] M. Simonovic, V. Nikolic, E. Petrovic, and I. Ciric, "Heat load prediction of small district heating system using artificial neural networks," Thermal Science, vol. 20, no. suppl. 5, pp. 1355-1365, 2016.

[7] S. Idowu, S. Saguna, C. Åhlund, and O. Schelén, "Applied machine learning: Forecasting heat load in district heating system," Energy and Buildings, vol. 133, pp. 478-488, Dec. 2016. [Online]. Available: http://www.sciencedirect.com/science/article/pii/S0378778816310155

[8] P. d. Saint-Aubain, "Adaptive Load Forecasting," 2011.

[9] P. Bacher, H. Madsen, and H. A. Nielsen, "Online short-term heat load forecasting for single family houses," in IECON Proceedings (Industrial Electronics Conference). IEEE, Nov. 2013, pp. 57415746.

[10] B. Talebi, P. A. Mirzaei, A. Bastani, and F. Haghighat, "A Review of District Heating Systems: Modeling and Optimization," Frontiers in Built Environment, vol. 2, 2016.

[11] P. Mattsson, D. Zachariah, and P. Stoica, "Recursive nonlinear-system identification using latent variables," Automatica, vol. 93, pp. 343-351, Jul. 2018.

[12] A. P. Dempster, N. M. Laird, and D. B. Rubin, "Maximum Likelihood from Incomplete Data Via the EM Algorithm," Journal of the Royal Statistical Society: Series B (Methodological), vol. 39, no. 1, pp. 1-22, Sep. 1977. [Online]. Available: http://doi.wiley.com/10.1111/j.2517-6161.1977.tb01600.x

[13] P. Stoica and P. Babu, "SPICE and LIKES: Two hyperparameter-free methods for sparse-parameter estimation," Signal Processing, vol. 92, no. 7, pp. 1580-1590, Jul. 2012.

[14] D. Romanchenko, J. Kensby, M. Odenberger, and F. Johnsson, "Thermal energy storage in district heating: Centralised storage vs. storage in thermal inertia of buildings," Energy Conversion and Management, vol. 162, pp. 26-38, Apr. 2018.

[15] I. Ballarini and V. Corrado, "Analysis of the building energy balance to investigate the effect of thermal insulation in summer conditions," Energy and Buildings, vol. 52, pp. 168-180, Sep. 2012.

[16] J. Gustafsson, J. Delsing, and J. van Deventer, "Thermodynamic Simulation of a Detached House with District Heating Subcentral," in 2008 2nd Annual IEEE Systems Conference. Montreal, QC, Canada: IEEE, Apr. 2008, pp. 1-8.

[17] H. Gadd and S. Werner, "Heat load patterns in district heating substations," Applied Energy, vol. 108, pp. 176-183, Aug. 2013. 